\title{
Electronic Synoptic Reporting of Thyroid Nodules: Potential for Reduction in Number of Patients Undergoing Thyroid Nodule Biopsies
}

\author{
Jimmy Tanche Wang1*, Paul Babyn1, Gary Groot², Rob Otani1 \\ ${ }^{1}$ Department of Radiology, University of Saskatchewan, Faculty of Medicine, Royal University of Hospital, \\ Saskatoon, Canada \\ ${ }^{2}$ Department of Surgery, University of Saskatchewan, Faculty of Medicine, Royal University of Hospital, \\ Saskatoon, Canada \\ Email: tanche.wang@usask.ca
}

Received 18 July 2016; accepted 29 August 2016; published 2 September 2016

Copyright (C) 2016 by authors and Scientific Research Publishing Inc.

This work is licensed under the Creative Commons Attribution International License (CC BY). http://creativecommons.org/licenses/by/4.0/

(c) (i) Open Access

\section{Abstract}

Purpose: The objective of the study was to design and implement an electronic synoptic report for thyroid sonography that incorporates the thyroid imaging reporting and data system (TIRADS) and assess potential for reducing unnecessary fine needle aspiration biopsies (FNAB) of thyroid nodules. Methods: The electronic synoptic report was developed using a relational database based on elements from TIRADS and a multidisciplinary consensus statement for thyroid reporting. A retrospective analysis of 138 patients with previously reported thyroid sonographic exams was evaluated for the presence of these elements. The electronic synoptic report calculates the TIRADS score and generates a formal report. Using the TIRADS score the potential decrease in unnecessary FNAB was estimated. Results: Key TIRADS elements were variously reported ranging from $43 \%$ for the thyroid nodule's architecture as solid or cystic. Thyroid nodule echogenicity and calcification was commented in $27 \%$ and $23 \%$, respectively. Other features of the TIRADS score were commented in $0 \%$ to $8 \%$ of the official reports. Estimated reduction for potentially reduced need for FNAB was $34.5 \%$. Conclusions: This study is the first implementation of synoptic reporting using a relational database for sonography of thyroid nodules. Implementation of an electronic standardized synoptic reporting system may facilitate more accurate, and more comprehensive reporting for thyroid ultrasound scanning of thyroid nodules. The use of TIRADS was estimated to be able to potentially reduce the need for FNAB which was significant.

\footnotetext{
${ }^{*}$ Corresponding author.
} 


\section{Keywords}

\section{Synoptic Reporting, Thyroid Nodules, Thyroid Cancer, Fine Needle Aspiration Biopsy, Thyroid Imaging Reporting and Data System}

\section{Introduction}

Palpable thyroid nodules occur at a rate of $5 \%$ in the general population [1] while thyroid sonography depicts nodules in up to $67 \%$ of the population [2]. The recent rise in cross-sectional imaging use especially CT and sonography has resulted in discovery of an increased number of asymptomatic thyroid nodules [3] with $5 \%-15 \%$ of these incidentally discovered thyroid nodules being malignant [1]. These numerous suspicious nodules have heightened demand for further investigations including biopsy and surgery to exclude malignancy, increased overall costs for the healthcare system and stimulated the search for better ways to reduce unnecessary biopsies and ensure that only appropriate cases go on to further biopsy or surgery. To better refine which nodules should undergo biopsy, scoring systems have been developed with the aim of increasing the likelihood of malignancy in the sampled population. One such system is the thyroid imaging reporting and data system also known as TIRADS [2], [4]. TIRADS stratifies thyroid malignancy risk by looking at the number of suspicious ultrasound features present [2]. Use of TIRADS may reduce unnecessary biopsies, surgeries and follow-up investigations [5]. Despite the apparent benefits of such scoring systems, the use of these scoring systems has been limited to date.

Synoptic reporting is rapidly gaining interest in various medical disciplines including pathology, clinical assessment, surgery and radiology as a way to improve communication and ensure completeness of reports. Documentation performed traditionally with a narrative dictated report may omit essential findings or critical components of a procedure. Failure to record specific details may be wrongly interpreted as negative findings rather than omissions.

Use of a structured or synoptic reporting format facilitates collection of important findings and also improves communications between clinicians, surgeons, pathologists and radiologists [6]. Electronic implementation of a synoptic reporting system provides many benefits including efficient documentation, automated report generation, and potential for data mining for research.

Use of a synoptic report for thyroid nodule sonography could ensure that all necessary elements of TIRADS are completed, and facilitate communication. Reporting of thyroid nodule and lymph node sonographic features is well suited for synoptic reporting as it involves a limited number of well-defined findings to be documented with standardized terminology [7]. With such an electronic synoptic reporting system a radiologist can efficiently document the important findings needed for report generation. With these data elements collected there is the potential to automatically calculate the TIRADS score and thereby provide improved communication to the referring clinician and surgeon.

We therefore proceeded to design and implement an electronic synoptic report at our institution. Data elements to be included were based on a multidisciplinary consensus statement on thyroid ultrasound reporting [7]. Based on the data inputted, the program calculates the TIRADS score and generates a formal report. Using retrospective data we subsequently tested the potential ability of our electronic synoptic reporting system to generate a TIRADS score and its potential to reduce unnecessary biopsies in our institution.

\section{Methods}

\subsection{Study Population}

Our institutional ethics review board approved this retrospective study and waived the requirement to obtain informed consent. All patients with thyroid sonography performed in the Saskatoon Health Region, over the period of January 2012 to May 2014 were included in this study if they underwent further thyroid biopsy or thyroid surgery.

\subsection{Creation of Synoptic Report}

\subsubsection{TIRADS}

We used a previously described and validated thyroid imaging reporting and data system (TIRADS) [2]. This 
scoring system was developed to stratify the risk of malignancy for thyroid nodules based on the presence or absence of a number of suspicious sonographic findings for malignancy [2], [4]. A simplified version of TIRADS [2], was used to stratify risk of malignancy for thyroid nodules (see Table 1). Suspicious ultrasound features for malignancy include solidity, hypoechogenicity or marked hypoechogenicity, microlobulated margins or irregular margins, microcalcification, and taller-than-wide shape. TIRADS 1 corresponds to a highly probable benign nodule (no suspicious US features), TIRADS 4A (one suspicious US feature) and 4B (two suspicious US feature) correspond to a low and intermediate suspicion for malignancy, respectively, TIRADS 4c is of moderate concern but not classic for malignancy (three or four suspicious US features), while TIRADS 5 (five suspicious US features) is highly suggestive of malignancy.

\subsubsection{Database}

An electronic synoptic report was designed based on a recently released multidisciplinary consensus statement for thyroid nodule reporting [7]. The Thyroid, Head and Neck Cancer Foundation convened a panel of nine specialists from a variety of disciplines involved in thyroid cancer to achieve a consensus on ultrasound evaluation of the thyroid gland, thyroid nodules, cervical lymph nodes, and fine needle aspiration of thyroid nodules and/or cervical lymph nodes. The included multidisciplinary consensus statement data fields are comprehensive and include all TIRADS elements. A relational database was used to implement the electronic synoptic report (Access 2013, Microsoft, Redmond, Washington). The created synoptic report provides information on the thyroid gland, nodule characteristics, cervical lymph nodes, and follow-up recommendations. The relational database calculates the thyroid imaging reporting and data system (TIRADS) score according to data entered [2]. The data elements collected with the database are shown in Figures 1-5. An automated sonography report is generated (Figure 6). To simplify the process, all the data fields are automatically set to default as normal values.

\subsubsection{Methodology of Review of Previously Reported Studies}

All included sonography examinations had been interpreted by board certified staff radiologists. The finalized formal radiologist reports of the thyroid ultrasounds were retrospectively reviewed for all needed characteristics for the thyroid gland, any thyroid nodules, or cervical lymph nodes as included in the TIRADS score and the multidisciplinary consensus statement for thyroid nodule reporting [7]. These formal elements of the thyroid gland and nodules were evaluated for their presence or absence in the prior official radiologist report. Any element not commented on the formal report was considered as not completed. The percentage of standard parameters completed was calculated.

\subsubsection{Estimation of the Potential Decrease in Unnecessary FNAB}

To estimate the potential decrease in unnecessary fine needle aspiration biopsy (FNAB) we utilized previous published recommendations [5]. Nodules that scored TIRADS 3 may be considered safe to monitor without FNAB unless they increase in size in a proven way. The number of nodules categorized as TIRADS 3 was compared to the number of nodules categorized as TIRADS 3 that were biopsied to estimate the decrease in FNAB with the application of TIRADS. Previously published data estimate the number of nodules that were likely to grow at 35\% [5]. This estimation of nodules that will grow and will need biopsies was multiplied by the number of nodules with a score of TIRADS 3 to estimate the decrease in unnecessary FNAB.

Table 1. TIRADS scoring system based on the number of suspicious malignant ultrasound features present including: solidity, hypoechogenicity or marked hypoechogenicity, microlobulated margins or irregular margins, microcalcification, and taller-than-wide shape [2].

\begin{tabular}{cccc}
\hline \# of Suspicious US Features & TIRADS Category & Risk & Probably Benign \\
0 & 3 & Low suspicion for malignancy \\
1 & $4 \mathrm{a}$ & Intermediate suspicion for malignancy \\
2 & $4 \mathrm{~b}$ & Moderate concern but not classis for malignancy \\
3 or 4 & $4 \mathrm{c}$ & Highly suggestive of malignancy \\
5 & 5 & 9.3 \\
\hline
\end{tabular}




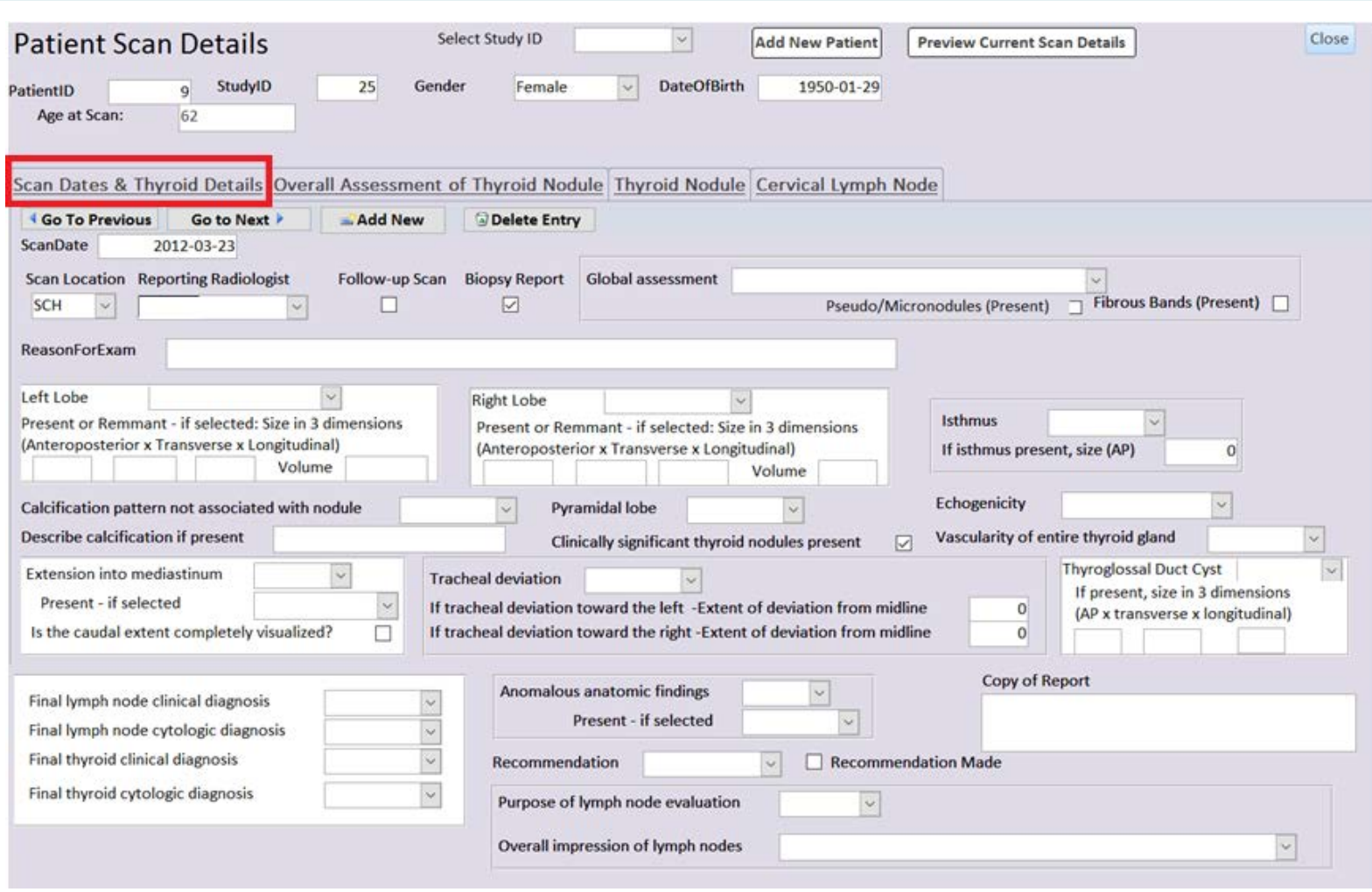

Figure 1. Screen 1 of relational database interface for thyroid gland labeled "scan dates \& thyroid details". Form to collect general information on the thyroid gland. Fields derived from multidisciplinary consensus statement [7]. General assessment of the thyroid gland notes if thyroid gland was normal, solitary nodule present, multinodular thyroid or diffuse thyroiditis. Final thyroid and lymph node diagnosis obtained from pathological report. Purpose of lymph node exams include screening for initial or follow-up ultrasound of uncharacterized nodule, preoperative mapping, or surveillance of thyroid cancer followup.

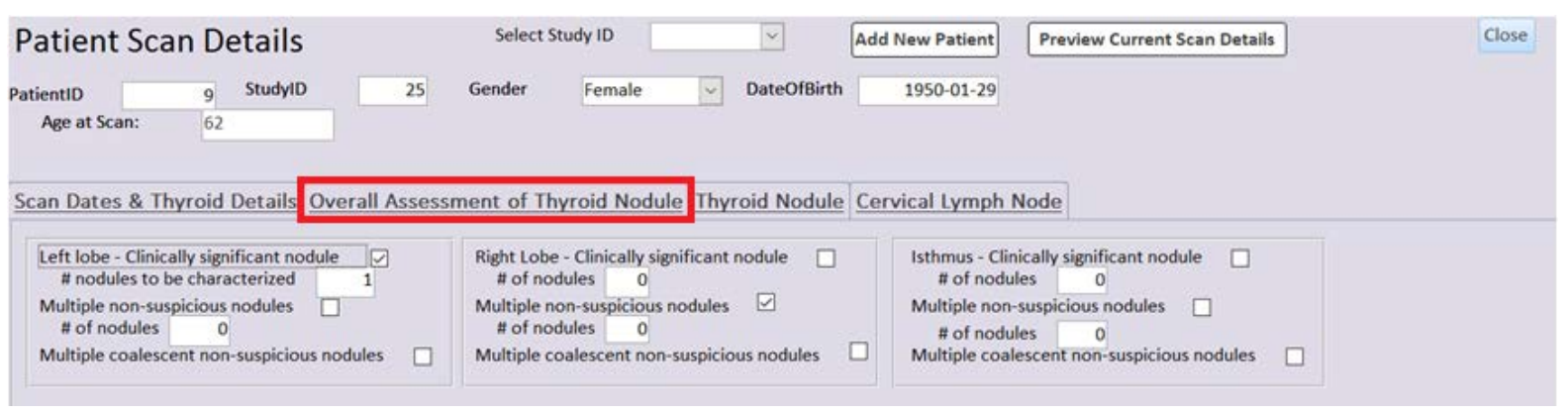

Figure 2. Screen 2 of relational database interface for overall assessment of each thyroid nodule labeled "overall assessment of thyroid nodule”. Collection of information regarding the number of nodules for each lobe and isthmus.

\section{Results}

\subsection{Study Population}

A total of 138 patients were included with all patients undergoing subsequent FNAB and 36 patients' thyroidectomy. 122 patients were female and 16 were male with patients ranging in age from 21 to 89 years (mean, 53.1 years). The mean age of male patients was 64 years (range, 42 - 82 years), and the mean age of female patients was 52.1 years (range, 21 - 88 years). The mean nodule size ( \pm standard deviation) was $1.83 \pm 1.38 \mathrm{~cm}$ (range, $0.1-12.5 \mathrm{~cm}$ ).

Total of 290 thyroid nodules were identified and US-guided fine needle aspiration biopsy (FNAB) was performed in 157 focal thyroid nodules. Of the 157 nodules biopsied, 103 were benign, 10 were malignant, and the 


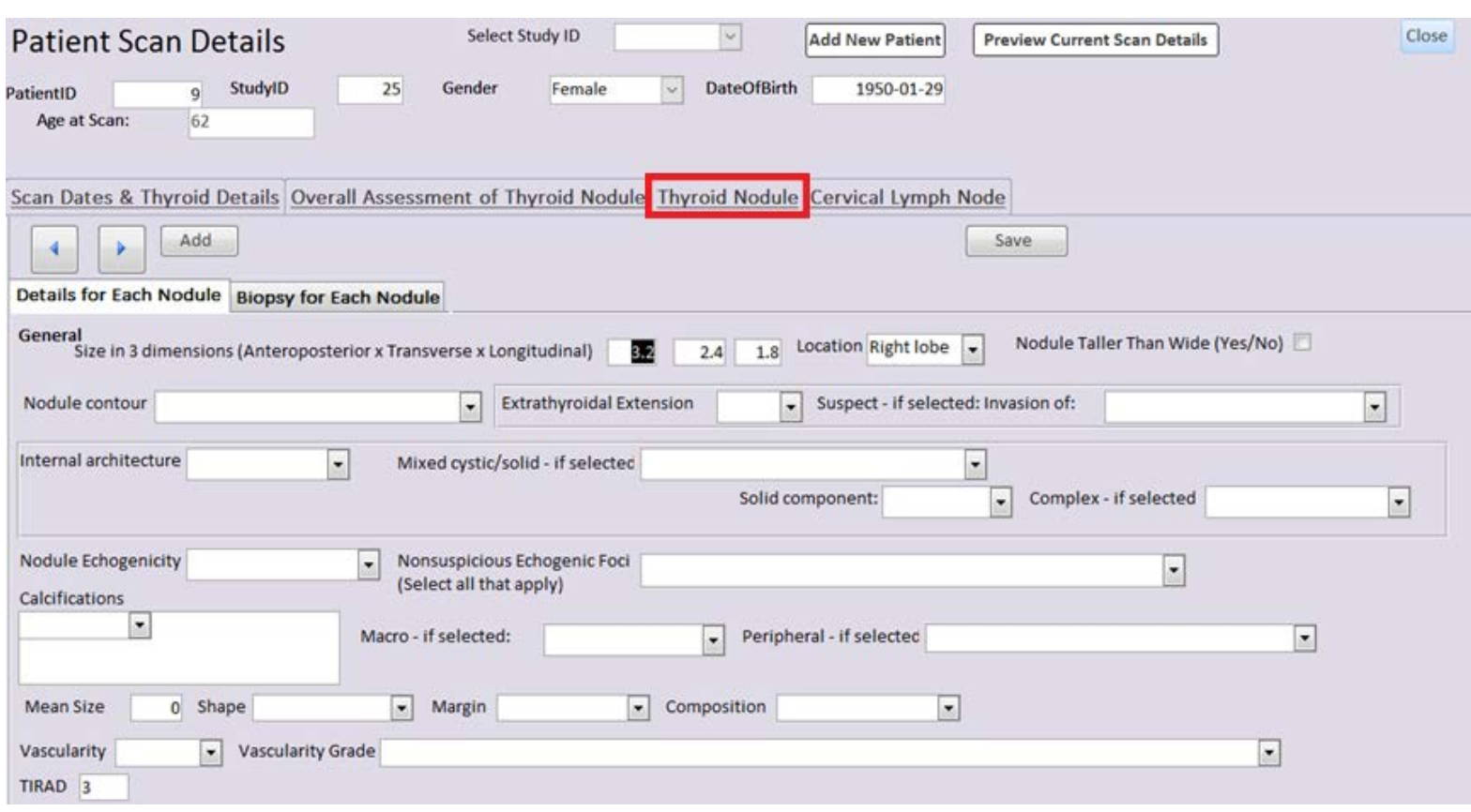

Figure 3. Screen 3 of relational database interface for individual details of each thyroid nodule labeled "thyroid nodule". *Data field containing descriptive suspicious features used in thyroid imaging reporting and data system (TIRADS) including solidity, hypoeechogenicity, microlobulated or irregular margins, microcalcification, and taller-than-wide shape.

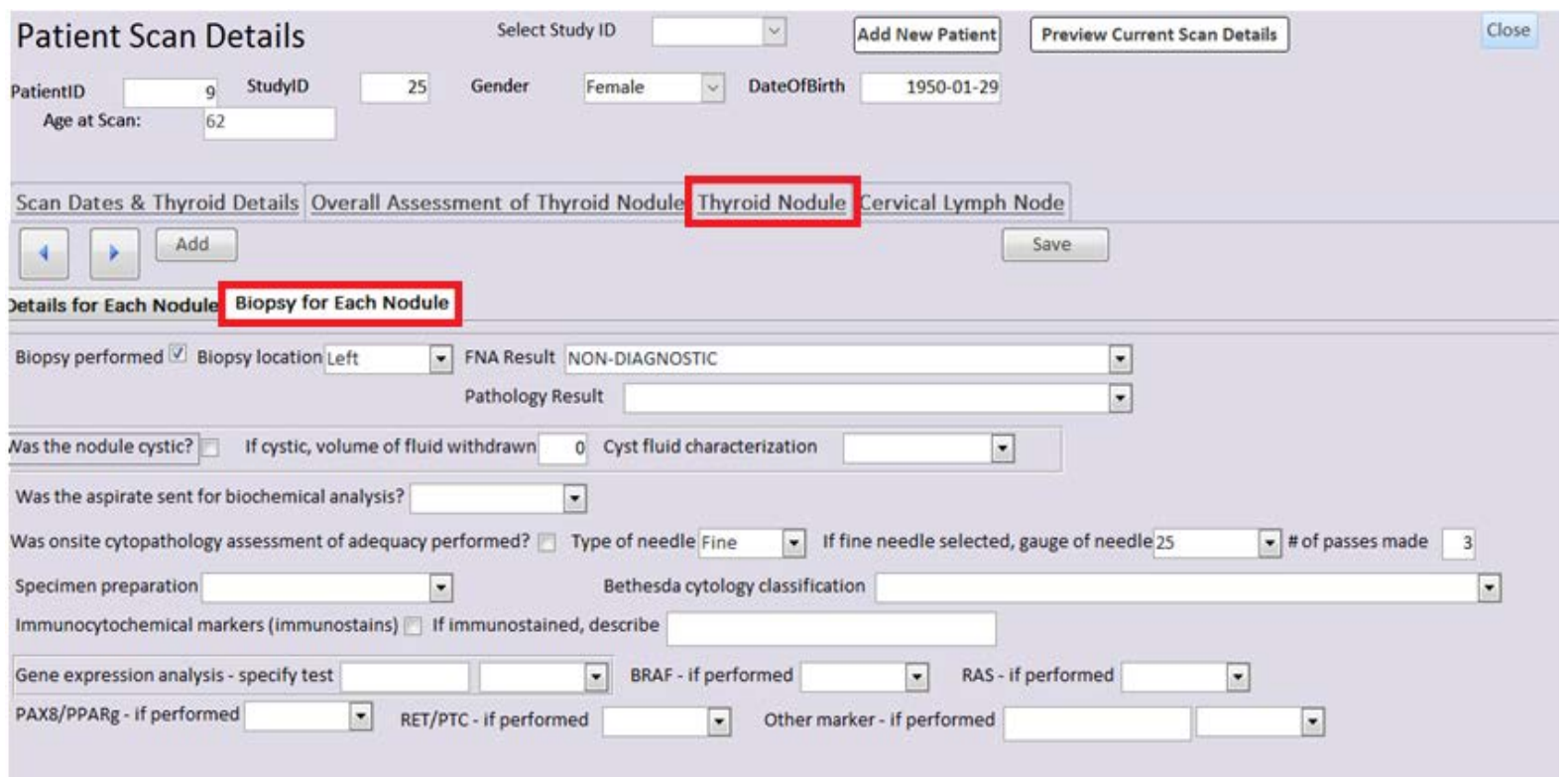

Figure 4. Embedded screen under screen 3 "thyroid nodule" in relational database interface for biopsy for each nodule labeled as "biopsy for each nodule". Technical details and results regarding the fine needle aspiration biopsy. Cytopathological tests ordered with the biopsy.

results for 44 nodules were non-diagnostic or unsatisfactory. Seventeen benign nodules and ten malignant nodules were confirmed with surgery. Eighty-six benign nodules were confirmed with cytologic examination (Figure 7). Three non-diagnostic nodules were surgically confirmed to be benign. Six nodules were considered unsatisfactory based on cytology of which three nodules were confirmed by surgery to be benign and three malignant. The pathologic diagnoses of the 36 thyroid nodules assessed by surgery are listed in Table 2 . There were 13 multinodular and 20 nodular thyroids on surgical pathology. 


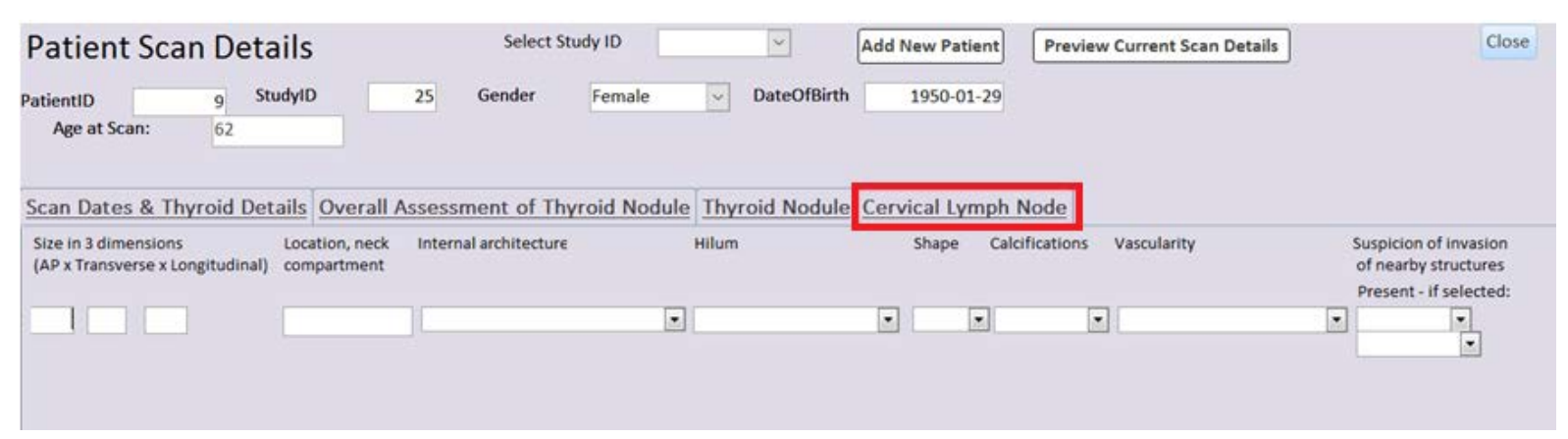

Figure 5. Screen 4 of relational database interface for cervical lymph nodes labeled as "cervical lymph node”. Details regarding the cervical lymph nodes including size, location, solid or cystic, presence of hilum, shape, presence of calcification, presence and location of vascularity, and presence of local invasion.

\section{Medical Imaging}

Patient: John Smith

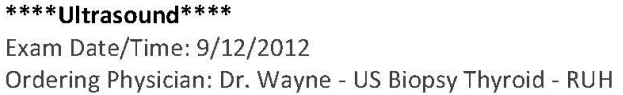

\section{CLINICAL INFORMATION}

Clinically palpable thyroid nodule

\section{COMPARISON}

Date of previous scan 2012-02-07.

\section{FINDINGS}

The left lobe is present and measures $1 \times 1 \times 1 \mathrm{~cm}$. The right lobe is present and measures $1.1 \times 2 \times 2 \mathrm{~cm}$. Isthmus is present and measures $0.4 \mathrm{~cm}$.

Clinically significant nodules are present. The left lobe contains 1 and the right lobe has 0 clinically significant nodules.

$$
\begin{aligned}
& \text { 2011-04-18 left lobe } 1 \text { The nodule on the left lobe has a completely smooth and well-defined contour } \\
& \text { and measures } 2.4 \times 1 \times 1 \mathrm{~cm} .
\end{aligned}
$$

\section{ULTRASOUND-GUIDED THYROID FINE NEEDLE ASPIRATION}

The benefits and risks of the procedure, including bleeding, infection, and injury to vascular and other structures were explained in detail, and the patient seemed to understand and provided informed consent.

The patient was supine, with her neck extended. The left anterior neck was marked, prepped, draped and anesthetized with $1 \%$ lidocaine plain $0.5 \mathrm{cc}$.

\section{BIOPSY FINDINGS}

Scan Date

2011-04-18

Using a 25 gauge needle 3 separate passes were made including with and without suction.

Post-biopsy scanning demonstrated no complication with no evidence of any hematoma. The patient was discharged in stable condition from the Medical Imaging Department.

\section{IMPRESSION:}

Thyroid with a nodule in the isthmus with TIRADS 3.

Uncomplicated ultrasound-guided, fine-needle aspiration of the dominant thyroid nodule.

\section{****FINAL REPORT****}

Principal interpreter: White.

Figure 6. Sample report automatically generated by relational database for thyroid nodule ultrasound and fine needle aspiration biopsy. 


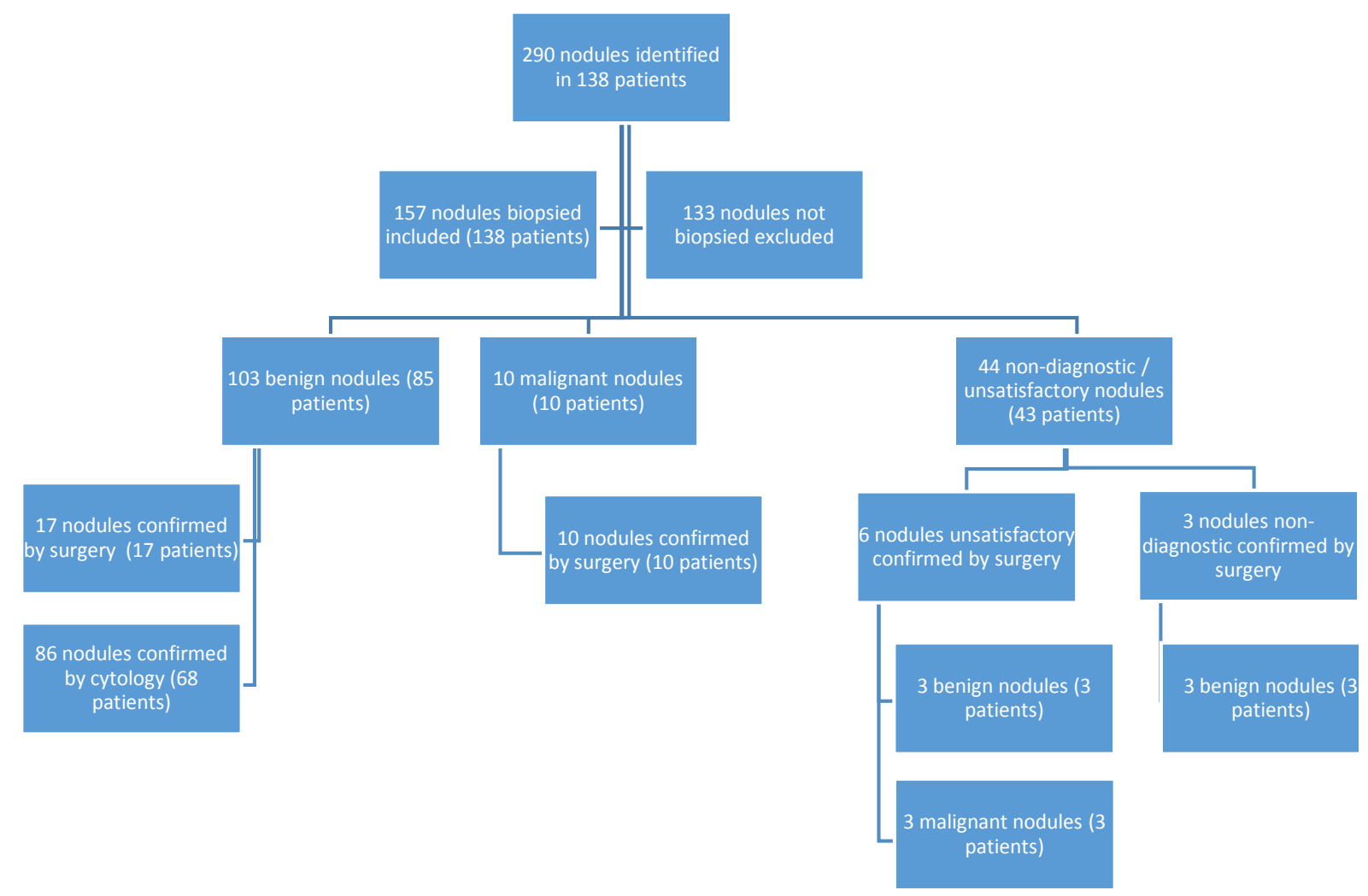

Figure 7. Summary of thyroid nodules used in the study.

Table 2. Histopathologic findings in the 36 nodules treated with surgery.

\begin{tabular}{|c|c|c|}
\hline Histopathologic Findings & No. of Nodules & $\%$ \\
\hline \multicolumn{3}{|l|}{ Benign $(n=26)$} \\
\hline ADENOMATOUS NODULE & 1 & 3.8 \\
\hline ATYPICAL FOLLICULAR ADENOMA & 2 & 7.7 \\
\hline BENIGN COLLOID NODULE & 2 & 7.7 \\
\hline BENIGN HÜRTHLE CELL ADENOMA & 1 & 3.8 \\
\hline FOLLICULAR ADENOMA & 1 & 3.8 \\
\hline GRANULOMATOUS THYROIDITIS & 1 & 3.8 \\
\hline HASHIMOTOS THYROIDITIS & 1 & 3.8 \\
\hline LYMPHOCYTIC THYROIDITIS & 1 & 3.8 \\
\hline MULTINODULAR THYROID & 4 & 15.4 \\
\hline NODULAR HYPERPLASIA OF THYROID & 12 & 46.2 \\
\hline \multicolumn{3}{|l|}{ Malignant $(\mathrm{n}=10)$} \\
\hline FOLLICULAR CARCINOMA & 1 & 10 \\
\hline HURTHLE CELL CARCINOMA & 1 & 10 \\
\hline PAPILLARY CARCINOMA & 7 & 70 \\
\hline PAPILLARY MICROCARCINOMA & 1 & 10 \\
\hline
\end{tabular}




\subsection{Synoptic Report}

The data fields collected in the synoptic report are divided into four main screens which are scan dates and thyroid details (Figure 1), overall assessment of thyroid nodule (Figure 2), thyroid nodule (Figure 3 and Figure 4) and cervical lymph node (Figure 5). Information on the thyroid gland includes global assessment of the thyroid gland (solitary nodule, multinodular, diffuse thyroiditis), dimensions of the thyroid lobes and isthmus, echogenicity, vascularity, and calcification (Figure 1). The overall assessment of the thyroid nodules includes the number of nodules in each lobe (Figure 2). Each clinically significant thyroid nodule is described in the thyroid nodule screen (Figure 3). Each clinically significant nodule is characterized by its location, dimensions, contour, internal architecture (cystic, solid, or mixed), echogenicity, calcifications, and vascularity. Cervical lymph nodes are described by their dimensions, location, internal architecture (solid or cystic), hilum, shape, calcifications, vascularity, and invasion of nearby structures (Figure 5). The purpose of the lymph node evaluation was recorded as brief survey for initial or follow-up ultrasound, preoperative detailed exam, or thyroid cancer follow-up. An overall impression of the lymph nodes as benign or suspicious was also recorded. A form collects technical details of the fine needle aspiration including needle size, number of passes, and cytopathological testing (Figure 4). Clicking on the "Preview Current Scan Details" button automatically generates a formal report for the thyroid nodules and biopsy report if applicable (Figure 6).

\subsection{Comparison of Official Report and TIRADS}

Figure 8 shows the percentages of formal elements of the thyroid nodules based on the multidisciplinary consensus statement that were completed in the official radiologist reports. Specific elements used in the TIRADS score are highlighted. Only $43 \%$ of official reports noted whether the thyroid nodule's architecture was solid or cystic. $27 \%$ of the reports commented on the thyroid nodule's echogenicity. $23 \%$ of the reports noted the calcification of the thyroid nodule. Other features of the TIRADS score such as taller-than-wide and nodule contour were commented in $0 \%$ and $8 \%$ of the official reports. TIRADS elements not included in the report were assumed to be absent in calculation of the TIRADS score.

\subsection{Estimation of the Decrease in Unnecessary FNAB}

Based on the data collected, an estimation of the reduction in the number of FNAB can be calculated. Of the 290 nodules, 154 nodules were categorized as TIRADS 3 representing $53.1 \%$ of all nodules. Ninety one of the nodules (63\%) categorized as TIRADS 3 were biopsied. Based on the reasoning of only $35 \%$ of nodules will increase in size in time, 66 nodules or $42 \%$ fewer nodules may have been spared from FNAB. Assuming an average

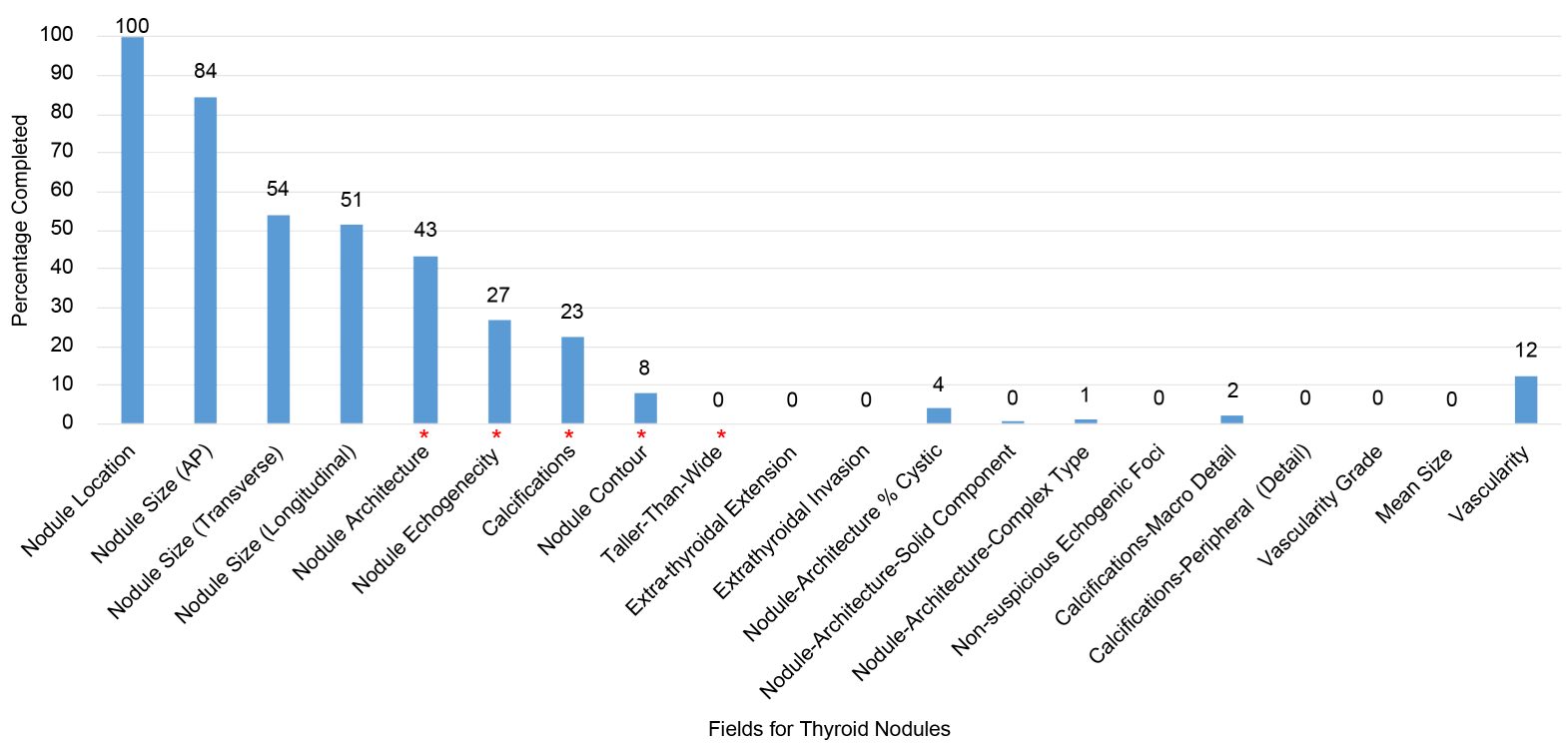

Figure 8. Completion rate of descriptive terminology abstracted from multidisciplinary consensus statement for thyroid nodules in ultrasound scans of the thyroid gland. *Descriptive suspicious feature used in TIRADS. 
of two nodules per patient, 34.5\% of patients (33 patients out of 97 patients) with TIRADS 3 may have been spared from FNAB with the application of TIRADS.

\section{Discussion}

At autopsy thyroid nodules are commonly discovered with over $50 \%$ of individuals without known thyroid disease having palpable or non-palpable nodules [8]. The widespread increased use of imaging has led to a higher detection rate of thyroid nodules and resulted in a significant increase in the number of thyroid FNAB being performed [2]. However less than $10 \%$ of these nodules are malignant [2]. Identifying suspicious malignant features on ultrasound better discerns the risk of malignancy and can guide subsequent management with fine needle aspiration biopsy (FNAB), sonographic follow-up, and need for surgical resection [9].

Avoiding unnecessary repeated US examinations and FNABs led to the development of risk stratification tools such as TIRADS [2], [4]. TIRADS provides standardized terminology to facilitate communication between clinicians, surgeons, pathologists and radiologists. Risk stratification based on TIRADS may provide management recommendations.

Synoptic reporting have been shown to be successful in increasing the completeness of operative reports [10], and improving the quality of surgical oncology [11] and pathology reports [6], [12]. Interest in synoptic reporting in radiology is developing [13]. Synoptic reporting may facilitate the comprehensive reporting of sonographic examinations of the thyroid gland and improve completeness of ultrasound reports. A synoptic report template should include all relevant findings on thyroid sonographic examinations. Synoptic reporting may be readily integrated into the workflow with potential to reduce the use of transcription services and improve turnaround time. Incorporation of logic into the synoptic report allows for calculations and grading to be performed automatically.

Linking structured reporting with a formalized reporting scheme is of obvious value. Using an electronic synoptic report facilitates the implementation of TIRADS by providing a standard documentation interface to include all the important sonographic features used in TIRADS and automatically generating the TIRADS score. Additional benefits include improved workflow and adherence to reporting guidelines and standards by automated sonographic report generation. The electronic system, secondarily acts as a research database for outcomes analysis and research. An electronic implementation of the standardized synoptic report may enhance efficiency, workflow, and patient care.

Our study showed a wide variation in ultrasound reporting of characteristics of thyroid nodules. No ultrasound report in our study mentioned all the ultrasound features linked with elevated risk of malignancy according to the TIRADS score. The percentage completed for the included sonographic features for the TIRADS score was poor. For taller-than-wide, nodule contour, nodule architecture, nodule echogenicity, and calcifications it was $0 \%, 8 \%, 43 \%, 27 \%$, and $23 \%$ respectively.

We estimated the potential decrease in unnecessary FNAB based on previous published material. In this study, 157 nodules were biopsied or $54.1 \%$ of the 290 . Sixty three percent of nodules (91 nodules) categorized as TIRAD 3 were biopsied. With the application of TIRADS we estimate a $42 \%$ reduction of biopsies needed with sparing of 66 nodules from FNAB. Our study suggests a greater reduction in the number of FNAB than suggested by the literature [5]. The literature suggests estimating the reduction in the number of FNAB based on a reasoning that $35 \%$ of nodules do not increase in size with time. Nodules with TIRADS scores of 3 represented $53.1 \%$ of all nodules in this study. Based on this reasoning, we deduce a 34.5\% $(0.65=$ score 3 that do not grow $\times$ 0.531 = percentage of nodules scored 3) estimated reduction in the number of FNAB.

Limitations of the current study are that data were collected retrospectively from sonographic reports. As not all fields were collected it may underestimate the effectiveness of TIRADS. Another limitation is that this is a single center study performed in a tertiary centre and may not be representative of all centers. However, despite its limitations, the estimated reduction in FNAB is likely underestimated and remains significant.

\section{Conclusion}

This study is the first implementation of synoptic reporting using a relational database for sonography of thyroid nodules. Implementation and use of an electronic standardized synoptic reporting system will facilitate more accurate, and more comprehensive reporting for thyroid ultrasound scanning of thyroid nodules. The use of TIRADS integrated into the electronic reporting system can help guide management and may lead to a reduction 
of the number of FNAB needed. Although retrospective, the use of TIRADS in our patient population was estimated to be able to potentially reduce the need for FNAB by $34.5 \%$ for this study.

\section{References}

[1] Nachiappan A.C., Metwalli, Z.A., Hailey, B.S., Patel, R.A., Ostrowski, M.L. and Wynne, D.M. (2014) The Thyroid: Review of Imaging Features and Biopsy Techniques with Radiologic-Pathologic Correlation. Radiographics, 34, 276 293. http://dx.doi.org/10.1148/rg.342135067

[2] Kwak, J.Y., Han, K.H., Yoon, J.H., Moon, H.J., Son, E.J., Park, S.H., et al. (2011) Thyroid Imaging Reporting and Data System for US Features of Nodules: A Step in Establishing Better Stratification of Cancer Risk. Radiology, 260, 892-899. http://dx.doi.org/10.1148/radiol.11110206

[3] Russ, G., Leboulleux, S., Leenhardt, L. and Hegedus, L. (2014) Thyroid Incidentalomas: Epidemiology, Risk Stratification with Ultrasound and Workup. European Thyroid Journal, 3, 154-163. http://dx.doi.org/10.1159/000365289

[4] Kwak, J.Y., Jung, I., Baek, J.H., Baek, S.M., Choi, N., Choi, Y.J., et al. (2013) Image Reporting and Characterization System for Ultrasound Features of Thyroid Nodules: Multicentric Korean Retrospective Study. Korean Journal of Radiology, 14, 110-117. http://dx.doi.org/10.3348/kjr.2013.14.1.110

[5] Russ, G., Royer, B., Bigorgne, C., Rouxel, A., Bienvenu-Perrard, M. and Leenhardt, L. (2013) Prospective Evaluation of Thyroid Imaging Reporting and Data System on 4550 Nodules with and without Elastography. European Journal of Endocrinology, 168, 649-655. http://dx.doi.org/10.1530/EJE-12-0936

[6] Parwani, A.V., Mohanty, S.K. and Becich, M.J. (2008) Pathology Reporting in the 21st Century: The Impact of Synoptic Reports and Digital Imaging. Laboratory Medicine, 39, 582-586. http://dx.doi.org/10.1309/LMAPF116QTKIDEBG

[7] Su, H.K., Dos Reis, L.L., Lupo, M.A., Milas, M., Orloff, L.A., Langer, J.E., et al. (2014) Striving toward Standardization of Reporting of Ultrasound Features of Thyroid Nodules and Lymph Nodes: A Multidisciplinary Consensus Statement. Thyroid, 24, 1341-1349. http://dx.doi.org/10.1089/thy.2014.0110

[8] Malhi, H., Beland, M.D., Cen, S.Y., Allgood, E., Daley, K., Martin, S.E., et al. (2014) Echogenic Foci in Thyroid Nodules: Significance of Posterior Acoustic Artifacts. American Journal of Roentgenology, 203, 1310-1316. http://dx.doi.org/10.2214/AJR.13.11934

[9] Perros, P., Boelaert, K., Colley, S., Evans, C., Evans, R.M., Gerrard, G.E., et al. (2014) Guidelines for the Management of Thyroid Cancer. Clinical Endocrinology (Oxford), 81, 1-122. http://dx.doi.org/10.1111/cen.12515

[10] Iyer, N.G., Nixon, I.J., Palmer, F., Ganly, I., Patel, S.G. and Shaha, A.R. (2011) Electronic synoptic Operative Reporting for Thyroid Surgery Using an Electronic Data Management System: Potential for Prospective Multicenter Data Collection. Annals of Surgical Oncology, 18, 762-766. http://dx.doi.org/10.1245/s10434-010-1361-0

[11] Chambers, A.J., Pasieka, J.L. and Temple, W.J. (2009) Improvement in the Accuracy of Reporting Key Prognostic and Anatomic Findings during Thyroidectomy by Using a Novel Web-Based Synoptic Operative Reporting System. Surgery, 146, 1090-1098. http://dx.doi.org/10.1016/j.surg.2009.09.032

[12] Tsan, C.J., Serpell, J.W. and Poh, Y.Y. (2007) The Impact of Synoptic Cytology Reporting on Fine-Needle Aspiration Cytology of Thyroid Nodules. ANZ Journal of Surgery, 77, 991-995. http://dx.doi.org/10.1111/j.1445-2197.2007.04297.x

[13] Tee, J.W., Chan, P.C., Gruen, R.L. and Rosenfeld, J.V. (2011) Synoptic Reporting for Spine Trauma. Spine (Phila Pa 1976), 36, 1997. http://dx.doi.org/10.1097/BRS.0b013e3182333089 


\section{Submit or recommend next manuscript to SCIRP and we will provide best service for you:}

Accepting pre-submission inquiries through Email, Facebook, LinkedIn, Twitter, etc.

A wide selection of journals (inclusive of 9 subjects, more than 200 journals)

Providing 24-hour high-quality service

User-friendly online submission system

Fair and swift peer-review system

Efficient typesetting and proofreading procedure

Display of the result of downloads and visits, as well as the number of cited articles

Maximum dissemination of your research work

Submit your manuscript at: http://papersubmission.scirp.org/ 